\title{
Integrins (version 2019.4) in the IUPHAR/BPS Guide to Pharmacology Database
}

Richard W. Farndale ${ }^{1}$ and Gavin E. Jarvis ${ }^{1}$

1. University of Cambridge, UK

\begin{abstract}
Integrins are unusual signalling proteins that function to signal both from the extracellular environment into the cell, but also from the cytoplasm to the external of the cell. The intracellular signalling cascades associated with integrin activation focus on protein kinase activities, such as focal adhesion kinase and Src. Based on this association between extracellular signals and intracellular protein kinase activity, we have chosen to include integrins in the 'Catalytic receptors' section of the database until more stringent criteria from NC-IUPHAR allows precise definition of their classification.
\end{abstract}

Integrins are heterodimeric entities, composed of $\alpha$ and $\beta$ subunits, each 1TM proteins, which bind components of the extracellular matrix or counter-receptors expressed on other cells. One class of integrin contains an inserted domain (I) in its $\alpha$ subunit, and if present (in $\alpha 1, \alpha 2, \alpha 10, \alpha 11, \alpha \mathrm{D}, \alpha \mathrm{E}, \alpha \mathrm{L}, \alpha \mathrm{M}$ and $\alpha \mathrm{X}$ ), this I domain contains the ligand binding site. All $\beta$ subunits possess a similar I-like domain, which has the capacity to bind ligand, often recognising the RGD motif. The presence of an $\alpha$ subunit I domain precludes ligand binding through the $\beta$ subunit. Integrins provide a link between ligand and the actin cytoskeleton (through typically short intracellular domains). Integrins bind several divalent cations, including a $\mathrm{Mg}^{2+}$ ion in the I or I-like domain that is essential for ligand binding. Other cation binding sites may regulate integrin activity or stabilise the 3D structure. Integrins regulate the activity of particular protein kinases, including focal adhesion kinase and integrin-linked kinase. Cellular activation regulates integrin ligand affinity via inside-out signalling and ligand binding to integrins can regulate cellular activity via outside-in signalling.

Several drugs that target integrins are in clinical use including: (1)abciximab ( $\alpha$ llb $\beta 3)$ for short term prevention of coronary thrombosis, (2) vedolizumab ( $\alpha 4 \beta 7)$ to reduce gastrointestinal inflammation, and (3) natalizumab $(\alpha 4 \beta 1)$ in some cases of severe multiple sclerosis.

\section{Contents}

This is a citation summary for Integrins in the Guide to Pharmacology database (GtoPdb). It exists purely as an adjunct to the database to facilitate the recognition of citations to and from the database by citation analyzers. Readers will almost certainly want to visit the relevant sections of the database which are given here under database links.

GtoPdb is an expert-driven guide to pharmacological targets and the substances that act on them. GtoPdb is a reference work which is most usefully represented as an on-line database. As in any publication this work should be appropriately cited, and the papers it cites should also be recognized. This document provides a 
citation for the relevant parts of the database, and also provides a reference list for the research cited by those parts.

Please note that the database version for the citations given in GtoPdb are to the most recent preceding version in which the family or its subfamilies and targets were substantially changed. The links below are to the current version. If you need to consult the cited version, rather than the most recent version, please contact the GtoPdb curators.

\section{Database links}

Integrins

http://www.guidetopharmacology.org/GRAC/FamilyDisplayForward?familyld=760

\section{Receptors}

Complexes

integrin $\alpha 1 \beta 1$

http://www.guidetopharmacology.org/GRAC/ObjectDisplayForward?objectld=2577

integrin $\alpha 2 \beta 1$

http://www.guidetopharmacology.org/GRAC/ObjectDisplayForward?objectld=2578

integrin $\alpha \mathrm{llb} \beta 3$

http://www.guidetopharmacology.org/GRAC/ObjectDisplayForward?objectld=2579

integrin $\alpha 4 \beta 1$

http://www.guidetopharmacology.org/GRAC/ObjectDisplayForward?objectld=2580

integrin $\alpha 4 \beta 7$

http://www.guidetopharmacology.org/GRAC/ObjectDisplayForward?objectld=2770

integrin $\alpha 5 \beta 1$

http://www.guidetopharmacology.org/GRAC/ObjectDisplayForward?objectld=2581

integrin $\alpha 6 \beta 1$

http://www.guidetopharmacology.org/GRAC/ObjectDisplayForward?objectld=2867

integrin $\alpha 10 \beta 1$

http://www.guidetopharmacology.org/GRAC/ObjectDisplayForward?objectld=2868

integrin $\alpha 11 \beta 1$

http://www.guidetopharmacology.org/GRAC/ObjectDisplayForward?objectld=2869

integrin $\alpha E \beta 7$

http://www.guidetopharmacology.org/GRAC/ObjectDisplayForward?objectld=2799

integrin $\alpha \mathrm{L} \beta 2$

http://www.guidetopharmacology.org/GRAC/ObjectDisplayForward?objectld=2582

integrin $\alpha \mathrm{V} \beta 3$

http://www.guidetopharmacology.org/GRAC/ObjectDisplayForward?objectld=2583

Receptors and Subunits

integrin, alpha 1 subunit

http://www.guidetopharmacology.org/GRAC/ObjectDisplayForward?objectld=2437

integrin, alpha 2 subunit (CD49B, alpha 2 subunit of VLA-2 receptor)

http://www.guidetopharmacology.org/GRAC/ObjectDisplayForward?objectld=2440

integrin, alpha Ilb subunit (platelet glycoprotein Ilb of Ilb/IIla complex, antigen CD41)

http://www.guidetopharmacology.org/GRAC/ObjectDisplayForward?objectld=2441

integrin, alpha 3 subunit (antigen CD49C, alpha 3 subunit of VLA-3 receptor)

http://www.guidetopharmacology.org/GRAC/ObjectDisplayForward?objectld=2442

integrin, alpha 4 subunit (antigen CD49D, alpha 4 subunit of VLA-4 receptor)

http://www.guidetopharmacology.org/GRAC/ObjectDisplayForward?objectld=2443

integrin, alpha 5 subunit (fibronectin receptor, alpha polypeptide)

http://www.guidetopharmacology.org/GRAC/ObjectDisplayForward?objectld=2444 
integrin, alpha 6 subunit

http://www.guidetopharmacology.org/GRAC/ObjectDisplayForward?objectld=2445

integrin, alpha 7 subunit

http://www.guidetopharmacology.org/GRAC/ObjectDisplayForward?objectld=2446

integrin, alpha 8 subunit

http://www.guidetopharmacology.org/GRAC/ObjectDisplayForward?objectld=2447

integrin, alpha 9 subunit

http://www.guidetopharmacology.org/GRAC/ObjectDisplayForward?objectld=2448

integrin, alpha 10 subunit

http://www.guidetopharmacology.org/GRAC/ObjectDisplayForward?objectld=2438

integrin, alpha 11 subunit

http://www.guidetopharmacology.org/GRAC/ObjectDisplayForward?objectld=2439

integrin, alpha D subunit

http://www.guidetopharmacology.org/GRAC/ObjectDisplayForward?objectld=2449

integrin, alpha E subunit (antigen CD103, human mucosal lymphocyte antigen 1; alpha polypeptide)

http://www.guidetopharmacology.org/GRAC/ObjectDisplayForward?objectld=2450

integrin, alpha L subunit (antigen CD11A (p180), lymphocyte function-associated antigen 1; alpha polypeptide)

http://www.guidetopharmacology.org/GRAC/ObjectDisplayForward?objectld=2451

integrin, alpha M subunit (complement component 3 receptor 3 subunit)

http://www.guidetopharmacology.org/GRAC/ObjectDisplayForward?objectld=2452

integrin, alpha $\mathrm{V}$ subunit

http://www.guidetopharmacology.org/GRAC/ObjectDisplayForward?objectld=2453

integrin, alpha $X$ subunit (complement component 3 receptor 4 subunit)

http://www.guidetopharmacology.org/GRAC/ObjectDisplayForward?objectld=2454

integrin, beta 1 subunit (fibronectin receptor, beta polypeptide, antigen CD29 includes MDF2, MSK12)

http://www.guidetopharmacology.org/GRAC/ObjectDisplayForward?objectld=2455

integrin, beta 2 subunit (complement component 3 receptor 3 and 4 subunit)

http://www.guidetopharmacology.org/GRAC/ObjectDisplayForward?objectld=2456

integrin, beta 3 subunit (platelet glycoprotein IIla, antigen CD61)

http://www.guidetopharmacology.org/GRAC/ObjectDisplayForward?objectld=2457

integrin, beta 4 subunit

http://www.guidetopharmacology.org/GRAC/ObjectDisplayForward?objectld=2458

integrin, beta 5 subunit

http://www.guidetopharmacology.org/GRAC/ObjectDisplayForward?objectld=2459

integrin, beta 6 subunit

http://www.guidetopharmacology.org/GRAC/ObjectDisplayForward?objectld=2460

integrin, beta 7 subunit

http://www.guidetopharmacology.org/GRAC/ObjectDisplayForward?objectld=2461

integrin, beta 8 subunit

http://www.guidetopharmacology.org/GRAC/ObjectDisplayForward?objectld=2462

\section{References}

1. Boehringer Ingelheim. LFA-1 (lymphocyte function-associated antigen-1) antagonist | BI-1950

2. Breitenstein W, Huerzeler M, Kelly T, Mancuso R, Schneider G and Weitz-Schmidt G. (2015) Small molecule Ifa-1 inhibitors Patent number: WO2015189265.

3. Coller BS and Knight DM. (1999) Method of antithrombotic therapy using anti-GPIlb/llla antibodies or fragments thereof, including c7E3. Patent number: US5976532.

4. Derkach DN, Wadekar SA, Perkins KB, Rousseau E, Dreiza CM, Cheung-Flynn J, Ramos HC, Ugarova TP and Sheller MR. (2010) RGD-dependent binding of TP508 to integrin alphavbeta3 mediates cell 
adhesion and induction of nitric oxide. Thromb. Haemost. 104: 172-82 [PMID:20508901]

5. Eldred CD, Evans B, Hindley S, Judkins BD, Kelly HA, Kitchin J, Lumley P, Porter B, Ross BC and Smith KJ et al.. (1994) Orally active non-peptide fibrinogen receptor (Gpllb/llla) antagonists: identification of 4-[4[4-(aminoiminomethyl)phenyl]-1-piperazinyl]-1-piperidineacetic acid as a long-acting, broad-spectrum antithrombotic agent. J. Med. Chem. 37: 3882-5 [PMID:7966149]

6. Fong S and Dennis MS.. (2009) Humanized anti-beta7 antagonists and uses therefor. Patent number: US7528236 B2.

7. Goodman SL, Hölzemann G, Sulyok GA and Kessler H. (2002) Nanomolar small molecule inhibitors for alphav(beta)6, alphav(beta)5, and alphav(beta)3 integrins. J. Med. Chem. 45: 1045-51 [PMID:11855984]

8. Han J, Liu S, Rose DM, Schlaepfer DD, McDonald H and Ginsberg MH. (2001) Phosphorylation of the integrin alpha 4 cytoplasmic domain regulates paxillin binding. J. Biol. Chem. 276: 40903-9 [PMID:11533025]

9. Hutchinson JH, Halczenko W, Brashear KM, Breslin MJ, Coleman PJ, Duong LT, Fernandez-Metzler C, Gentile MA, Fisher JE and Hartman GD et al.. (2003) Nonpeptide alphavbeta3 antagonists. 8. In vitro and in vivo evaluation of a potent alphavbeta3 antagonist for the prevention and treatment of osteoporosis. J. Med. Chem. 46: 4790-8 [PMID:14561098]

10. Jardieu PM and Presta LG. (2004) Method of treatment using humanized anti-CD11a antibodies. Patent number: US6703018.

11. Kelly TA, Jeanfavre DD, McNeil DW, Woska Jr JR, Reilly PL, Mainolfi EA, Kishimoto KM, Nabozny GH, Zinter R and Bormann BJ et al.. (1999) Cutting edge: a small molecule antagonist of LFA-1-mediated cell adhesion. J. Immunol. 163: 5173-7 [PMID:10553036]

12. Kishimoto TK, Hollander N, Roberts TM, Anderson DC and Springer TA. (1987) Heterogeneous mutations in the beta subunit common to the LFA-1, Mac-1, and p150,95 glycoproteins cause leukocyte adhesion deficiency. Cell 50: 193-202 [PMID:3594570]

13. Lee Y, Kang DK, Chang SI, Han MH and Kang IC. (2004) High-throughput screening of novel peptide inhibitors of an integrin receptor from the hexapeptide library by using a protein microarray chip. $J$ Biomol Screen 9: 687-94 [PMID:15634795]

14. Liles WC, Dale DC, Price TH, Gaviria JM, Turner T, Saoud J and Frumkin LR. (2000) Inhibition of in vivo neutrophil transmigration by a novel humanized anti-CD11/CD18 monoclonal antibody. Cytokines Cell. Mol. Ther. 6: 121-6 [PMID:11140880]

15. Lin Kc, Ateeq HS, Hsiung SH, Chong LT, Zimmerman CN, Castro A, Lee WC, Hammond CE, Kalkunte S and Chen LL et al.. (1999) Selective, tight-binding inhibitors of integrin alpha4beta1 that inhibit allergic airway responses. J. Med. Chem. 42: 920-34 [PMID:10072689]

16. Liu G, Link JT, Pei Z, Reilly EB, Leitza S, Nguyen B, Marsh KC, Okasinski GF, von Geldern TW and Ormes $M$ et al.. (2000) Discovery of novel p-arylthio cinnamides as antagonists of leukocyte functionassociated antigen-1/intracellular adhesion molecule-1 interaction. 1. Identification of an additional binding pocket based on an anilino diaryl sulfide lead. J. Med. Chem. 43: 4025-40 [PMID:11052808]

17. Marcinkiewicz C, Weinreb PH, Calvete JJ, Kisiel DG, Mousa SA, Tuszynski GP and Lobb RR. (2003) Obtustatin: a potent selective inhibitor of alpha1beta1 integrin in vitro and angiogenesis in vivo. Cancer Res. 63: 2020-3 [PMID:12727812]

18. Matsuno H, Stassen JM, Vermylen J and Deckmyn H. (1994) Inhibition of integrin function by a cyclic RGD-containing peptide prevents neointima formation. Circulation 90: 2203-6 [PMID:7955174]

19. Miller MW, Basra S, Kulp DW, Billings PC, Choi S, Beavers MP, McCarty OJ, Zou Z, Kahn ML and Bennett JS et al.. (2009) Small-molecule inhibitors of integrin alpha2beta1 that prevent pathological thrombus formation via an allosteric mechanism. Proc. Natl. Acad. Sci. U.S.A. 106: 719-24 [PMID:19141632]

20. No authors listed. (2004) Natalizumab: AN 100226, anti-4alpha integrin monoclonal antibody.Drugs $R D$ 5: 102-7 [PMID:15293871]

21. Ponath PD, Ringler DJ, ST, Newman W, Saldanha J and Bendig MM. (2006) Humanized immunoglobulin reactive with $\alpha 4 \beta 7$ integrin. Patent number: US7147851 B1. 
22. Scarborough RM and Gretler DD. (2000) Platelet glycoprotein Ilb-IIla antagonists as prototypical integrin blockers: novel parenteral and potential oral antithrombotic agents. J. Med. Chem. 43: 3453-73 [PMID:10999999]

23. Soler D, Chapman T, Yang LL, Wyant T, Egan R and Fedyk ER. (2009) The binding specificity and selective antagonism of vedolizumab, an anti-alpha4beta7 integrin therapeutic antibody in development for inflammatory bowel diseases. J. Pharmacol. Exp. Ther. 330: 864-75 [PMID:19509315]

24. Trstenjak U, Ilaš J and Kikelj D. (2013) Low molecular weight dual inhibitors of factor Xa and fibrinogen binding to GPIIb/IIla with highly overlapped pharmacophores. Eur J Med Chem 64: 302-13 [PMID:23644213]

25. Wu H and Gao C. (2010) Eph receptor Fc variants with enhanced antibody dependent cell-mediated cytotoxicity activity. Patent number: US7659374.

26. Yao N, Xiao W, Wang X, Marik J, Park SH, Takada Y and Lam KS. (2009) Discovery of targeting ligands for breast cancer cells using the one-bead one-compound combinatorial method. J. Med. Chem. 52: 12633 [PMID:19055415]

27. Yasuda T, Gold HK, Kohmura C, Guerrero L, Yaoita H, Fallon JT, Bunting S and Collen D. (1993) Intravenous and endobronchial administration of G4120, a cyclic Arg-Gly-Asp-containing platelet GPIIb/IIla receptor-blocking pentapeptide, enhances and sustains coronary arterial thrombolysis with rt-PA in a canine preparation. Arterioscler. Thromb. 13: 738-47 [PMID:8485125]

28. Yenari MA, Kunis D, Sun GH, Onley D, Watson L, Turner S, Whitaker S and Steinberg GK. (1998) Hu23F2G, an antibody recognizing the leukocyte CD11/CD18 integrin, reduces injury in a rabbit model of transient focal cerebral ischemia. Exp. Neurol. 153: 223-33 [PMID:9784282]

29. Zhong M, Gadek TR, Bui M, Shen W, Burnier J, Barr KJ, Hanan EJ, Oslob JD, Yu CH and Zhu Jet al.. (2012) Discovery and Development of Potent LFA-1/ICAM-1 Antagonist SAR 1118 as an Ophthalmic Solution for Treating Dry Eye. ACS Med Chem Lett 3: 203-6 [PMID:24900456] 